\title{
Bifurcations in a delayed fractional model of glucose-insulin interaction with incommensurate orders
}

\author{
Natchapon Lekdee ${ }^{1}$, Sekson Sirisubtawee ${ }^{1,2^{*}}$ (D) and Sanoe Koonprasert ${ }^{1,2}$ (D)
}

\author{
"Correspondence: \\ sekson.s@sci.kmutnb.ac.th \\ 'Department of Mathematics, \\ Faculty of Applied Science, King \\ Mongkut's University of Technology \\ North Bangkok, Bangkok, Thailand \\ ${ }^{2}$ Centre of Excellence in \\ Mathematics, CHE, Bangkok, \\ Thailand
}

\begin{abstract}
This paper proposes a delayed fractional-order model of glucose-insulin interaction in the sense of the Caputo fractional derivative with incommensurate orders. This fractional-order model is developed from the first-order model of glucose-insulin interaction. Firstly, we investigate the non-negativity and the boundedness of solutions of the fractional-order model. Secondly, the stability and the bifurcation of the model are studied by separating the associated characteristic equation of the model into its real and imaginary parts and taking a time delay as the bifurcation parameter. The asymptotic stability and the Hopf bifurcation are discussed via the condition of creation of the bifurcation. Furthermore, it is shown that the onset of the bifurcation is related to the fractional orders of the model. Finally, some numerical simulations of the model using the Adam-Bashforth-Moulton predictor corrector scheme are demonstrated to support our obtained theoretical results.
\end{abstract}

Keywords: Hopf bifurcation; Glucose-Insulin; Fractional model; Time delay; Stability analysis

\section{Introduction}

Several mathematicians have developed mathematical models describing the relationship between insulin and glucose in human beings. Such models have been restricted to integer-order ordinary differential equations and delay differential equations [1-5]. The most widely used model in physiological research on the metabolism of glucose is the socalled minimal model. In 1981, Bergman et al. [6] proposed a three-compartment minimal model to analyze and estimate insulin sensitivity and pancreatic responsivity to the glucose tolerance of human subjects. In 1986, Pacini and Bergman [7] applied the model to MINMOD, which is a computer program for the identification of model parameters for each individual. Gaetano and Arino [8] modified the minimal model by incorporating the insulin dynamics. Their modified model consists of the following three state variables: $G(t)$ denotes the plasma glucose concentration at time $t, I(t)$ represents the insulin concentration at time $t$, and $X(t)$ is an auxiliary function describing insulin-excitable tissue glucose uptake activity. The modified minimal model can be expressed as

$$
\frac{d G(t)}{d t}=-\left(p_{1}+X(t)\right) G(t)+p_{1} G_{b}
$$

(c) The Author(s) 2019. This article is distributed under the terms of the Creative Commons Attribution 4.0 International License (http://creativecommons.org/licenses/by/4.0/), which permits unrestricted use, distribution, and reproduction in any medium, provided you give appropriate credit to the original author(s) and the source, provide a link to the Creative Commons license, and indicate if changes were made. 


$$
\begin{aligned}
& \frac{d X(t)}{d t}=-p_{2} X(t)+p_{3}\left(I(t)-I_{b}\right), \\
& \frac{d I(t)}{d t}=p_{4}\left(G(t)-p_{5}\right)^{+} t-p_{6}\left(I(t)-I_{b}\right),
\end{aligned}
$$

with the initial conditions $G(0)=p_{0}, X(0)=0, I(0)=p_{7}+I_{b}$. The meaning of the parameters in the model, i.e., $p_{0}, p_{1}, p_{2}, \ldots, p_{7}, G_{b}$, and $I_{b}$ are defined in [8]. The term $\left(G(t)-p_{5}\right)^{+}$is taken to be $\left(G(t)-p_{5}\right)$ if $G(t)>b_{5}$, otherwise it is taken to be zero. However, there are three drawbacks for minimal model (1) as mentioned [8,9]. The first disadvantage of system (1) is that it does not admit an equilibrium point and its solutions may not be bounded. Since the minimal model cannot conduct a single-step parameter fitting process, then erratic estimates of insulin sensitivity definitely occur. This is considered as the second defect of the model. The final drawback of system (1) is that it does not have an explicit time delay to impede the action of insulin on glucose, but it performs an artificial delay via the variable $X(t)$ instead. Therefore, the authors in [8] introduced the dynamical model to solve the mentioned problems of the minimal model. However, the physiological hypotheses underlying Eq. (1) are still maintained; in other words, the disappearance of glucose from plasma may be explained as a first-order process of the rate which is both dependent and independent upon insulin concentration. The new model takes the form of

$$
\begin{aligned}
& \frac{d G(t)}{d t}=-b_{1} G(t)-b_{4} I(t) G(t)+b_{7}, \\
& \frac{I(t)}{d t}=-b_{2} I(t)+\frac{b_{6}}{b_{5}} \int_{t-b_{5}}^{t} G(s) d s .
\end{aligned}
$$

The initial conditions for the above model are $G(0)=G_{b}+b_{0}, I(0)=I_{b}+b_{3} b_{0}$, and $G(t) \equiv G_{b}$ for $t \in\left[-b_{5}, 0\right)$. Also, the meaning of the parameters in dynamical model (2), i.e., $b_{0}, b_{1}, b_{2}, \ldots, b_{7}, G_{b}$, and $I_{b}$, are described in [8]. However, the dynamical model gives a few assumptions that may not be necessary or realistic [9] as follows. For instance, the interaction term $b_{4} I(t) G(t)$ is too restrictive so it can be replaced by the more general and realistic term $\frac{b_{4} I(t) G(t)}{(\alpha G(t)+1)}$, where $\frac{1}{\alpha}$ denotes the half-saturation constant. Moreover, the approach used to introduce the delay $b_{5}$ in Eq. (2) is quite restrictive because it depends on only one subjective assumption that the effective pancreatic secretion of insulin at time $t$ is considered to be proportional to the average value of glucose concentration in the $b_{5}$ minutes preceding time $t$. Other possible ways of incorporating the time delay should be brought to fix this problem. Moreover, the model is always globally asymptotically stable. The main objective for a use of the above two models is only to study metabolism of glucose via the medical experiment called the intra venous glucose tolerance test (IVGTT) $[7,10]$.

Later on, Li and Kuang [9] thus generalized the dynamical model using some techniques, e.g., finding an appropriate way of incorporating time delay so that the new models can have unstable positive steady states and generate sustainable oscillatory solutions. In general, diabetic patients can exhibit intrinsic glucose oscillation [11]. Obtaining sustained insulin and glucose oscillations depends on two essential factors $[9,11,12]$, i.e., (i) a time delay of 30-45 min for the effect of insulin on hepatic glucose production and (ii) an inert effect of insulin on glucose utilization because insulin performs from a compartment remote from plasma. Obviously, both minimal model (1) and dynamical model (2) do not account for the delayed influence of insulin on the hepatic glucose production and are 
Table 1 Meaning of the parameters used in the model of glucose-insulin interaction in Eq. (3)

\begin{tabular}{ll}
\hline Parameter & Meaning \\
\hline$a_{1}$ & Insulin-independent constant of tissue glucose uptake rate \\
$a_{2}$ & Apparent first-order disappearance rate constant for insulin \\
$a_{3}$ & Constant measuring the insulin-dependent glucose disappearance rate per unit [pM] of \\
& plasma insulin concentration \\
$a_{4}$ & Reciprocal of the half-saturation constant of plasma glucose \\
$a_{5}$ & Second-phase pancreatic insulin release rate constant in mg/dl of the average plasma glucose \\
& concentration throughout the previous $\tau$ minutes \\
$a_{6}$ & Constant increase rate in plasma glucose concentration due to constant baseline liver glucose \\
$\tau$ & release \\
& Number of minutes of the past period whose plasma glucose concentrations influence the \\
\end{tabular}

not designed to explain insulin oscillation. Hence, these two models cannot produce unstable steady states. This is confirmed from the clinic data reported in [8]. Renaming the parameters of the specific model proposed by Li and Kuang with the ordered new parameters, the convenient model, which is the special case of their delay differential models of glucose-insulin interaction, can be written as follows [9]:

$$
\begin{aligned}
& \frac{d G(t)}{d t}=-a_{1} G(t)-\frac{a_{3} I(t) G(t)}{\left(a_{4} G(t)+1\right)}+a_{6}, \\
& \frac{d I(t)}{d t}=-a_{2} I(t)+a_{5} G(t-\tau),
\end{aligned}
$$

where $\tau \geq 0$ and the initial conditions are $G(t) \equiv G_{0}$ for $t \in[-\tau, 0]$ and $I(0)=I_{0}$. The above model in Eq. (3) consists of two differential equations describing the variation of blood glucose concentration $G(t)[\mathrm{mg} / \mathrm{dl}]$ and blood insulin concentration $I(t)[\mu \mathrm{UI} / \mathrm{ml}]$. The meaning of the positive parameters $a_{1}, a_{2}, a_{3}, a_{4}, a_{5}, a_{6}$, and $\tau$ are given in Table 1 . They provided two important differences of model (3) comparing with model (2). First, they used the more general and realistic Michaelis-Menten form $\frac{I(t) G(t)}{\left(a_{4} G(t)+1\right)}$ for insulindependent net glucose tissue uptake instead of $I(t) G(t)$. This is because of the capacity of insulin's ability for digesting glucose and the limit of time. Second, they assumed that the effective pancreatic secretion at time $t$ is affected by the value of glucose concentration in the $\tau$ minutes preceding time $t$ instead of the average amount in that period. Performing extensive simulations using the clinic data in [8], they successfully obtained periodic solutions in model (3). They concluded that even without hepatic glucose production, their model can give oscillatory solutions.

Recently, fractional-order differential equations, which are used to model complex phenomena, have been extensively applied in many fields [13-16]. This is because the behaviors of many biological systems have memory or hereditary properties which can be better described using fractional-order derivatives [17]. Hence, fractional-order differential equations, which use fractional-order derivatives such as the Caputo fractional derivative [18] and the Grünwald-Letnikov derivative [19], can be used to model real problems better than using integer-order differential equations for some particular problems. In consequence, several mathematicians and scientific researchers have introduced many fractional-order differential equation models developed from their original integer-order differential equation models.

There have been some articles describing the use of fractional-order differential equations to model real-world problems in mathematical biosciences. For example, Ertürk et 
al. [20] proposed and solved numerically the fractional-order differential system for a giving up smoking model using the multi-step generalized differential transform method (MSGDTM). Cho et al. [21] developed a fractional-order version of the minimal model for glucose-insulin dynamics. They also investigated local stability of the model. Carvalho and Pinto [22] proposed a delay fractional-order model for co-infection of malaria and HIV/AIDS while personal protection and vaccination against malaria are considered. Huang et al. [23] studied a bifurcation control for a novel incommensurate fractionalorder predator-prey system with a time delay. Lekdee et al. [24] solved a fractional-order model of the glucose-insulin homeostasis in rats, obtaining analytical and numerical solutions using the Laplace-Adomian-Padé method and the Adams-Bashforth-Moulton type predictor-corrector scheme. They also found all exact solutions of this fractionalorder model using the Laplace transform. More recent applications of fractional-order differential equations in biological studies can be found in [25-28].

In particular, many glucose-insulin interactions have been investigated using first-order differential equation models (see, e.g., [8, 11, 29-35]). However, there were some recent research evidences of the significant usefulness of fractional-order derivatives in constructing glucose-insulin models. For instance, in 2017, Sakulrang et al. [36] verified that deterministic fractional-order models could provide better fits than first-order differential equation models to continuous glucose monitoring (CGM) data from subjects with type 1 diabetes. They also concluded that for physiologic plausibility, their fractional-order models provide a reasonable rate of movement of glucose from the blood into the environment. In 2015, Kim et al. [21] proposed a generalized MINMOD Millennium model using the Caputo fractional-order derivative of order $\alpha \in(0,1]$. They discovered from the model that the value of $\beta^{1-\alpha}$ is inversely proportional to the value of $S_{I}$, where $\beta$ is a fractional time constant for preserving units and $S_{I}$ is the insulin sensitivity. This can be biologically interpreted as meaning that the concentrations of glucose and insulin in the system can be balanced by increasing the active rate $\beta^{1-\alpha}$ and decreasing $S_{I}$, or vice versa. This is because the value of $\beta^{1-\alpha}$ (e.g., $\beta^{1-\alpha}=1$ represents normal subjects while $\beta^{1-\alpha}=2$ is for diabetic patients) can be physiologically considered as the effect of the rheological behavior in enhancing the muscular and liver sensitivity to the action of insulin [6,37]. Hence, fractionalorder differential equations currently play a significant role in generalizing integer-order mathematical models for glucose-insulin dynamics so that some mentioned advantages of fractional-order derivatives are carried on with the classical models.

Motivated by the significance and importance of fractional-order derivatives and the advantages of the generalized dynamical model (3) as described above, we will convert the first-order delay differential equations for glucose-insulin interaction in Eq. (3) into a delayed fractional model in the sense of the Caputo fractional derivative with incommensurate orders. We will find a positive equilibrium point of the resulting system and analyze asymptotic stability of the system. In addition, we will establish conditions for which the fractional-order system undergoes a Hopf bifurcation at the equilibrium point. Finally, the Adams-Bashforth-Moulton type predictor-corrector method will be used to obtain numerical solutions of this model to confirm the theoretical analysis.

The paper is organized as follows. In Sect. 2, we present some preliminaries including the definition of Caputo fractional derivative and its properties and the asymptotic stability theorem for a delayed fractional differential system. The description of the modified Adams-Bashforth method for solving delayed fractional differential equations is con- 
tained in this section. In Sect. 3, the transformation from Eq. (3) to an incommensurate fractional-order differential system for glucose-insulin interaction is obtained. The nonnegativity and boundedness of solutions of the proposed model are proved in Sect. 4. The stability analysis of a positive equilibrium point of the resulting model is discussed in Sect. 5. The existence of a Hopf bifurcation for the model is established in this section. In Sect. 6, numerical simulations are provided. Finally, the conclusions are given in the last section.

\section{Preliminaries}

Frequently, real-world problems can be mathematically modeled using differential equations equipped with some initial conditions. Thus, Caputo's definition of fractional-order derivative is appropriate to replace the classical derivative used in the original models. In addition, the Laplace transform of Caputo's derivative can be written in terms of the given initial conditions of the problems and Caputo's derivative of a constant is equal to zero. Hence, we will reasonably use this fractional-order derivative for our delayed fractionalorder glucose-insulin interaction model.

A function $f(t)(t>0)$ is said to be in the space $C_{q}(q \in \mathbb{R})$ if it can be expressed as $f(t)=t^{p} g(t)$ for some $p>q$, where $g(t)$ is continuous in $[0, \infty)$. The function is also said to be in the space $C_{q}^{m}$ if $f^{(m)} \in C_{q}, m \in \mathbb{N}$ (see $[17,38]$ and the references cited therein for further details).

Definition 2.1 ([17]) The Riemann-Liouville fractional integral operator of order $q>0$ of a function $f \in C_{q}$ with $a \geq 0$ is defined as

$$
\mathrm{RL} J_{a}^{q} f(t)=\frac{1}{\Gamma(q)} \int_{a}^{t}(t-\tau)^{q-1} f(\tau) d \tau, \quad t>a,
$$

where $\Gamma(z)=\int_{0}^{\infty} e^{-u} u^{z-1} d u$ is the gamma function.

Definition 2.2 ([17]) For a positive real number $q$, the Caputo fractional derivative of order $q$ with $a \geq 0$ is defined in terms of the Riemann-Liouville fractional integral, i.e., ${ }_{C} D_{a}^{q} f(t)={ }_{\mathrm{RL}} J_{a}^{m-q} f^{(m)}(t)$, or it can be expressed as

$$
{ }_{C} D_{a}^{q} f(t)= \begin{cases}\frac{1}{\Gamma(m-q)} \int_{a}^{t} \frac{f^{(m)}(\tau)}{(t-\tau)^{q-m+1}} d \tau, & m-1<q<m, \\ \frac{d^{m}}{d t^{m}} f(t), & q=m,\end{cases}
$$

where $t \geq a, f \in C_{-1}^{m}$, and $m \in \mathbb{N}$. In particular, when $0<q \leq 1$, we have

$$
{ }_{C} D_{a}^{q} f(t)=\frac{1}{\Gamma(1-q)} \int_{a}^{t} \frac{f^{\prime}(\tau)}{(t-\tau)^{q}} d \tau
$$

Corollary 2.1 ([39]) Let $0<q \leq 1$. Suppose $f \in C[a, b]$ and ${ }_{C} D_{a}^{q} f \in(a, b]$. If ${ }_{C} D_{a}^{q} f(t) \geq 0$ for all $t \in(a, b)$, then the function $f$ is non-decreasing, and if ${ }_{C} D_{a}^{q} f(t) \leq 0$ for all $t \in(a, b)$, then the function $f$ is non-increasing.

Lemma 2.1 (Fractional comparison principle in [40]) Let $x(0)=y(0)$ and ${ }_{C} D_{a}^{q} x(t) \leq$ ${ }_{C} D_{a}^{q} y(t)$, where $0<q \leq 1$. Then $x(t) \leq y(t)$. 
The Laplace transforms of the Caputo fractional derivative and some types of the Mittag-Leffler functions are as follows.

Lemma 2.2 ([17]) The Laplace transform of the Caputo fractional derivative of order $m$ $1<q<m$ is

$$
\mathscr{L}\left\{{ }_{C} D_{a}^{q} f(t)\right\}=s^{q} F(s)-\sum_{k=0}^{m-1} s^{q-k-1} f^{(k)}(a)
$$

where $F(s)=\mathscr{L}\{f(t)\}$.

Definition 2.3 ([17]) The single parameter Mittag-Leffler function is defined by

$$
E_{q}(t)=\sum_{k=0}^{\infty} \frac{t^{k}}{\Gamma(q k+1)}, \quad q>0
$$

and the two parameter Mittag-Leffler function can be defined by

$$
E_{q, \beta}(t)=\sum_{k=0}^{\infty} \frac{t^{k}}{\Gamma(q k+\beta)}, \quad q, \beta>0
$$

It is not difficult to see that $E_{q, 1}(t)=E_{q}(t)$.

Lemma 2.3 ([17]) The Laplace transforms for several Mittag-Leffler functions are given by

$$
\begin{aligned}
& \mathscr{L}\left\{E_{q}\left(-\lambda t^{q}\right)\right\}=\frac{s^{q-1}}{s^{q}+\lambda}, \\
& \mathscr{L}\left\{t^{\beta-1} E_{q, \beta}\left(-\lambda t^{q}\right)\right\}=\frac{s^{q-\beta}}{s^{q}+\lambda},
\end{aligned}
$$

provided that $s>|\lambda|^{1 / q}$, where $\lambda$ is a constant parameter.

Definition 2.4 Consider the following fractional-order system:

$$
{ }_{C} D_{a}^{q} X(t)=F(X(t))
$$

where $X(t)=\left(x_{1}(t), x_{2}(t), \ldots, x_{n}(t)\right)^{T}, F(t)=\left(f_{1}(t), f_{2}(t), \ldots, f_{n}(t)\right)^{T}$, and $q=\left(q_{1}, q_{2}, \ldots, q_{n}\right)^{T}$ with $q_{i}>0, i=1,2, \ldots, n$. The equilibrium solution $X^{*}=\left(x_{1}^{*}, x_{2}^{*}, \ldots, x_{n}^{*}\right)^{T}$ of the system is defined by $F\left(X^{*}\right)=0$, i.e., $f_{i}\left(X^{*}\right)=0, i=1,2, \ldots, n$.

Consider a general delayed fractional-order system

$$
{ }_{C} D_{a}^{q} X(t)=F(X(t), X(t-\tau)),
$$

where $\tau>0$ is a time delay and $X(t)=\left(x_{1}(t), x_{2}(t), \ldots, x_{n}(t)\right)^{T} \in \mathbb{R}^{n}$. The equilibrium point $X^{*}$ of system (13) is the solution of the equation $F(X, X)=0$. The associated linearized system of system (13) at an equilibrium point $X^{*}$ can be written as

$$
{ }_{C} D_{a}^{q} U(t)=A U(t)+B U(t-\tau)
$$


where $A, B \in \mathbb{R}^{n \times n}$. The characteristic equation of system (14) is

$$
\Delta(s)=\operatorname{det}\left(s^{q} I-A-B e^{-s \tau}\right)=0,
$$

where $I \in \mathbb{R}^{n \times n}$ is the identity matrix. If $\tau=0$, the linearized system (14) is reduced to

$$
{ }_{C} D_{a}^{q} U(t)=M U(t)
$$

where the coefficient matrix $M=A+B$.

In $[23,41]$, the Hopf bifurcation conditions were investigated for the general delayed fractional-order system (13). Suppose that the following conditions hold, then system (13) encounters a Hopf bifurcation at the equilibrium $X^{*}$ when $\tau=\tau_{0}$.

(1) All the eigenvalues $\lambda_{i}, i=1,2,3, \ldots, n$, of the coefficient matrix $M$ of the linearized system of Eq. (13) satisfy the condition $\left|\arg \left(\lambda_{i}\right)\right|>\frac{q \pi}{2}, i=1,2,3, \ldots, n$.

(2) The characteristic equation $\Delta(s)=0$ in Eq. (15) has a pair of purely imaginary roots $\pm i \omega_{0}$ when $\tau=\tau_{0}$.

(3) $\operatorname{Re}\left[\frac{d s}{d \tau}\right]_{\tau=\tau_{0}} \neq 0$, where $\operatorname{Re}(\cdot)$ denotes the real part of a complex number.

Next the algorithm for solving a delayed fractional-order differential equation is briefly discussed as follows. Bhalekar and Daftardar-Gejji have modified the Adams-Bashforth method to solve delay differential equations of fractional order (FDDE) [42]. The method is described below. Consider the FDDE

$$
\begin{aligned}
& { }_{C} D_{a}^{q} y(t)=f(t, y(t), y(t-\tau)), \quad t \in[0, T], 0<q \leq 1, \\
& y(t)=g(t), \quad t \in[-\tau, 0] .
\end{aligned}
$$

For convenience, we use the starting point $a=0$. Then the Volterra integral equation of (17) can be written as

$$
y(t)=g(0)+\frac{1}{\Gamma(\alpha)} \int_{0}^{t}(t-\xi)^{\alpha-1} f(\xi, y(\xi), y(\xi-\tau)) d \xi
$$

Similarly, we obtain a uniform grid for the delayed problem as $\left\{t_{n}=n h: n=-k,-k+\right.$ $1, \ldots,-1,0,1, \ldots, N\}$, where $k$ and $N$ are integers such that $h=T / N$ and $h=\tau / k$. For the sake of simplicity, we let

$$
y_{h}\left(t_{j}\right)=g\left(t_{j}\right), \quad j=-k,-k+1, \ldots,-1,0,
$$

and note that

$$
y_{h}(t-\tau)=y_{h}(j h-k h)=y_{h}\left(t_{j-k}\right), \quad j=0,1, \ldots, N .
$$

We assume that the approximations $y_{h}\left(t_{j}\right) \approx y\left(t_{j}\right)$ for $j=-k,-k+1, \ldots,-1,0,1, \ldots, n$ have been calculated, and we want to calculate $y_{h}\left(t_{n+1}\right)$ using the formula

$$
y\left(t_{n+1}\right)=g(0)+\frac{1}{\Gamma(\alpha)} \int_{0}^{t+1}\left(t_{n+1}-\xi\right)^{\alpha-1} f(\xi, y(\xi), y(\xi-\tau)) d \xi
$$


Applying the product trapezoidal quadrature formula to approximate the integral in (22), by substituting approximations $y_{h}\left(t_{n}\right)$ for $y\left(t_{n}\right)$, we obtain

$$
\begin{aligned}
y_{h}\left(t_{n+1}\right)= & g(0)+\frac{h^{\alpha}}{\Gamma(\alpha+2)} f\left(t_{n+1}, y_{h}\left(t_{n+1}\right), y_{h}\left(t_{n+1}-\tau\right)\right) \\
& +\frac{h^{\alpha}}{\Gamma(\alpha+2)} \sum_{j=0}^{n} a_{j, n+1} f\left(t_{j}, y_{h}\left(t_{j}\right), y_{h}\left(t_{j}-\tau\right)\right) \\
= & g(0)+\frac{h^{\alpha}}{\Gamma(\alpha+2)} f\left(t_{n+1}, y_{h}\left(t_{n+1}\right), y_{h}\left(t_{n+1-k}\right)\right) \\
& +\frac{h^{\alpha}}{\Gamma(\alpha+2)} \sum_{j=0}^{n} a_{j, n+1} f\left(t_{j}, y_{h}\left(t_{j}\right), y_{h}\left(t_{j-k}\right)\right),
\end{aligned}
$$

where

$$
a_{j, n+1}= \begin{cases}n^{\alpha+1}-(n-\alpha)(n+1)^{\alpha} & \text { if } j=0, \\ (n-j+2)^{\alpha+1}+(n-j)^{\alpha+1}-2(n-j+1)^{\alpha+1}, & \text { if } 1 \leq j \leq n, \\ 1, & \text { if } j=n+1 .\end{cases}
$$

The product rectangle rule is then applied in (23) to evaluate the following predictor term:

$$
\begin{aligned}
y_{h}^{P}\left(t_{n+1}\right) & =g(0)+\frac{1}{\Gamma(\alpha)} \sum_{j=0}^{n} b_{j, n+1} f\left(t_{j}, y_{h}\left(t_{j}\right), y_{h}\left(t_{j}-\tau\right)\right) \\
& =g(0)+\frac{1}{\Gamma(\alpha)} \sum_{j=0}^{n} b_{j, n+1} f\left(t_{j}, y_{h}\left(t_{j}\right), y_{h}\left(t_{j-k}\right)\right),
\end{aligned}
$$

where

$$
b_{j, n+1}=\frac{h^{\alpha}}{\alpha}\left((n+1-j)^{\alpha}-(n-j)^{\alpha}\right) .
$$

\section{Fractional-order model description}

In this section, we develop a delayed fractional-order glucose-insulin interaction model, which is based on the generalized dynamical model (3), using the Caputo fractional derivative. Since model (3) is the delay differential system in which the derivative of $I(t)$ at time $t$ is given in terms of the value of glucose concentration in the $\tau$ minutes preceding time $t$, i.e., $G(t-\tau)$. The Caputo fractional-order derivative, which will be inserted into each equation of Eq. (3), contains the accumulated information of weighted ordinary derivatives from the starting point to the present time depending upon the value of its fractional order. Replacing the integer-order derivative in a classical delay differential system with the Caputo fractional-order derivative, we can utilize accumulative information of the rate of change of unknown functions appearing in the system besides only the values of the unknown functions at the current and previous times. Hence, the mathematical models constructed using fractional-order derivatives can express memory, history, or nonlocal effects. Furthermore, the theoretical and numerical results contained in [9] confirmed that the first-order model (3) can perform the following behaviors: asymptotically stable steady 
state, oscillatory solutions converging to the steady state, and sustainable oscillatory solutions when the values of the parameter $a_{4}$ and the delay $\tau$ are adjusted. In particular, if $a_{4}>0$ and $\tau$ is small, then the positive steady state becomes stable, while if $a_{4}$ is relatively small but $\tau$ is large, then oscillatory solutions, i.e., periodic solutions, may occur. This consequently invites us to study a fractional-order version of the generalized dynamical model (3) so that we can investigate whether fractional orders of the new model are an additional factor affecting the appropriate delays, which are required for the existence of a stable equilibrium or periodic solutions of the fractional model. If stability regions of the fractional-order model can be changed by its fractional orders, then by a mathematical point of view we can utilize this benefit for certain purposes. For example, calculating the suitable time delay $\tau$ depending on fractional orders of the system such that the concentrations of glucose and insulin in diabetic patients exhibit their asymptotically stable or sustainable oscillatory behaviors. In terms of physiological aspects, the fractional-order model of (3) could influence the delay action between insulin in plasma and its effect on the hepatic glucose production and also on a period range of oscillation in human insulin secretion. These cannot be obtained from using the first-order system (3). Therefore, this motivates us to develop a fractional-order version of the generalized dynamical model.

Replacing the first-order derivatives in Eq. (3) by the Caputo fractional derivatives of orders $q_{1}, q_{2} \in(0,1]$, respectively, the resulting equations still have the problem in which the units of the left-hand side and the right-hand side of the resulting system mismatch, i.e., the units of the left-hand side are time $e^{q_{1}}$ and time ${ }^{q_{2}}$ while the units of the right-hand side of the system have the dimension time ${ }^{-1}$. Hence, we must preserve units as described in [21, 43 ] on both sides of each equation in the resulting system by adjusting some of the original parameters or multiplying some original parameters by a fractional time constant $\beta$. After taking care of the units for both sides of the equations, we obtain the delayed fractionalorder model of glucose-insulin interaction with incommensurate orders as follows:

$$
\begin{aligned}
& { }_{C} D_{a}^{q_{1}} G(t)=-a_{1}^{q_{1}} G(t)-\frac{\beta^{1-q_{1}} a_{3} I(t) G(t)}{\left(a_{4} G(t)+1\right)}+\beta^{1-q_{1}} a_{6}, \\
& { }_{C} D_{a}^{q_{2}} I(t)=-a_{2}^{q_{2}} I(t)+\beta^{1-q_{2}} a_{5} G(t-\tau) .
\end{aligned}
$$

For convenience, we set

$$
\begin{aligned}
& \alpha_{1}=a_{1}^{q_{1}}, \quad \alpha_{2}=a_{2}^{q_{2}}, \quad \alpha_{3}=\beta^{1-q_{1}} a_{3}, \\
& \alpha_{4}=a_{4}, \quad \alpha_{5}=\beta^{1-q_{2}} a_{5}, \quad \alpha_{6}=\beta^{1-q_{1}} a_{6} .
\end{aligned}
$$

Then system (27) becomes

$$
\begin{aligned}
& { }_{C} D_{a}^{q_{1}} G(t)=-\alpha_{1} G(t)-\frac{\alpha_{3} I(t) G(t)}{\left(\alpha_{4} G(t)+1\right)}+\alpha_{6}, \\
& { }_{C} D_{a}^{q_{2}} I(t)=-\alpha_{2} I(t)+\alpha_{5} G(t-\tau),
\end{aligned}
$$

where $t \in[-\tau, T), \tau \geq 0$ and $T$ is some positive number. The initial conditions of system (29) are the same as those of system (3). It can be noticed that all new parameters, except $\alpha_{4}$, depend significantly on the fractional orders $q_{1}, q_{2}$ and the fractional time constant $\beta$. These new parameters maintain the biological meaning of the old parameters which they 
are associated with. However, their values can be varied via the values of $q_{1}, q_{2}$, and $\beta$ as set above. Especially, we call the parameters $\alpha_{1}$ and $\alpha_{2}$ the fractional tissue glucose uptake rate and the fractional disappearance rate constant for insulin, respectively. As mentioned in the generalized MINMOD Millennium model [21], the terms $\beta^{1-q_{1}}$ and $\beta^{1-q_{2}}$ can be physiologically considered as the parameters describing the rheological behavior in enhancing the muscular and liver sensibility to the action of insulin. Roughly speaking, the new fractional-order model (29) is the generalization of the first-order system (3).

The fractional-order system (29) will be used as our model for analysis and simulation in the following sections. In order to find equilibrium points of system (29), we $\operatorname{set}{ }_{C} D_{a}^{q_{1}} G(t)=$ 0 and ${ }_{C} D_{a}^{q_{2}} I(t)=0$ and then solve the obtained equations for an equilibrium point. It is not difficult to figure out that system (29) has a unique positive equilibrium point $\left(G^{*}, I^{*}\right)$, where

$$
\begin{aligned}
& G^{*}=\frac{\left(\alpha_{4} \alpha_{6}-\alpha_{1}+\sqrt{\left(\alpha_{4} \alpha_{6}-\alpha_{1}\right)^{2}+4\left(\alpha_{4} \alpha_{1}+\frac{\alpha_{3} \alpha_{5}}{\alpha_{2}}\right) \alpha_{6}}\right)}{2\left(\alpha_{4} \alpha_{1}+\frac{\alpha_{3} \alpha_{5}}{\alpha_{2}}\right)}, \\
& I^{*}=\frac{\alpha_{5}}{\alpha_{2}} G^{*} .
\end{aligned}
$$

In the following sections, the investigation of non-negative and bounded solutions of system (29) will be provided. Furthermore, the issue of a Hopf bifurcation for the proposed fractional-order system will be carried out when the time delay $\tau$ is used as a bifurcation parameter.

\section{Non-negative and bounded solutions}

In this section, we show the non-negativity and boundedness of the solutions of system (29) with some initial conditions. Denoting $R_{+}^{2}=\left\{(x, y) \in R^{2} \mid x, y \geq 0\right\}$, we have the following theorem.

Theorem 4.1 All solutions of the initial value problem, which is given by system (29) and the initial conditions $G(t) \equiv G_{0}$ for $t \in[-\tau, 0]$ and $I(0)=I_{0}$, exist for $t>0$. The solutions remain in $R_{+}^{2}$ and are bounded.

Proof Let $(G(t), I(t))$ be a solution of system (29). Assume that there exists a first time $t_{1}$ such that $G\left(t_{1}\right)=0$ and ${ }_{C} D_{a}^{q_{1}} G\left(t_{1}\right) \leq 0$. However, from the first equation of system (29), we have

$$
{ }_{C} D_{a}^{q_{1}} G\left(t_{1}\right)=-\alpha_{1} G\left(t_{1}\right)-\frac{\alpha_{3} I\left(t_{1}\right) G\left(t_{1}\right)}{\left(\alpha_{4} G\left(t_{1}\right)+1\right)}+\alpha_{6}=\alpha_{6}>0 .
$$

This contradiction shows that $G(t)>0$ for all $t>t_{1}$ by Corollary 2.1 .

Similarly, we assume that there exists a first time $t_{2}$ such that $I\left(t_{2}\right)=0$ and ${ }_{C} D_{a}^{q_{2}} I\left(t_{2}\right) \leq 0$. From the second equation of system (29), we then obtain

$$
{ }_{C} D_{a}^{q_{2}} I\left(t_{2}\right)=-\alpha_{2} I\left(t_{2}\right)+\alpha_{5} G\left(t_{2}-\tau\right)=\alpha_{5} G\left(t_{2}-\tau\right) .
$$

Since $G\left(t_{2}-\tau\right)>0$, this contradiction shows that $I(t)>0$ for all $t>t_{2}$ using Corollary 2.1. Hence, the solution of system (29) stays in $R_{+}^{2}$. 
As for the boundedness of $G(t)$, by the first equation of model (29), we have

$$
{ }_{C} D_{a}^{q_{1}} G(t) \leq-\alpha_{1} G(t)+\alpha_{6} .
$$

Let ${ }_{C} D_{a}^{q_{1}} G_{1}(t)=-\alpha_{1} G_{1}(t)+\alpha_{6}$ and $G_{1}(0)=G(0)$. Consider the following fractional differential equation:

$$
{ }_{C} D_{a}^{q_{1}} G_{1}(t)=-\alpha_{1} G_{1}(t)+\alpha_{6} .
$$

Taking the Laplace transform of Eq. (32) by using Eq. (7), we then obtain

$$
\begin{aligned}
& s^{q_{1}} \mathcal{G}_{1}(s)-s^{q_{1}-1} G_{1}(0)=-\alpha_{1} \mathcal{G}_{1}(s)+\frac{\alpha_{6}}{s}, \\
& \left(s^{q_{1}}+\alpha_{1}\right) \mathcal{G}_{1}(s)=\frac{\alpha_{6}}{s}+s^{q_{1}-1} G_{1}(0), \\
& \mathcal{G}_{1}(s)=\frac{\alpha_{6}}{s\left(s^{q_{1}}+\alpha_{1}\right)}+G_{1}(0) \frac{s^{q_{1}-1}}{s^{q_{1}}+\alpha_{1}},
\end{aligned}
$$

where $\mathcal{G}_{1}(s)=\mathscr{L}\left\{G_{1}(t)\right\}$. Taking the inverse Laplace transform of Eq. (33), we then have

$$
\begin{aligned}
G_{1}(t) & =\alpha_{6} \mathscr{L}^{-1}\left\{\frac{1}{s\left(s^{q_{1}}+\alpha_{1}\right)}\right\}+G_{1}(0) \mathscr{L}^{-1}\left\{\frac{s^{q_{1}-1}}{s^{q_{1}}+\alpha_{1}}\right\} \\
& =\alpha_{6} \mathscr{L}^{-1}\left\{\frac{s^{-1}}{\left(s^{q_{1}}+\alpha_{1}\right)}\right\}+G_{1}(0) \mathscr{L}^{-1}\left\{\frac{s^{q_{1}-1}}{s^{q_{1}}+\alpha_{1}}\right\} \\
& =\alpha_{6} \mathscr{L}^{-1}\left\{\frac{s^{q_{1}-\left(q_{1}+1\right)}}{\left(s^{q_{1}}+\alpha_{1}\right)}\right\}+G_{1}(0) \mathscr{L}^{-1}\left\{\frac{s^{q_{1}-1}}{s^{q_{1}}+\alpha_{1}}\right\} .
\end{aligned}
$$

Applying Eqs. (10) and (11) to Eq. (34), we have

$$
G_{1}(t)=\alpha_{6} t^{q_{1}} E_{q_{1}, q_{1}+1}\left(-\alpha_{1} t^{q_{1}}\right)+G_{0} E_{q_{1}, 1}\left(-\alpha_{1} t^{q_{1}}\right)
$$

where $E_{q_{1}, \beta}(z)$ is the two-parameter Mittag-Leffler function defined in Eq. (9). By Lemma 2.1, we now have

$$
0 \leq G(t) \leq G_{1}(t)=\alpha_{6} t^{q_{1}} E_{q_{1}, q_{1}+1}\left(-\alpha_{1} t^{q_{1}}\right)+G_{0} E_{q_{1}, 1}\left(-\alpha_{1} t^{q_{1}}\right), \quad \forall t>0 .
$$

Next we consider the terms $E_{q_{1}, 1}\left(-\alpha_{1} t^{q_{1}}\right)$ and $t^{q_{1}} E_{q_{1}, q_{1}+1}\left(-\alpha_{1} t^{q_{1}}\right)$ in Eq. (35) as $t \rightarrow \infty$. We must then consider the asymptotic behavior of the Mittag-Leffler functions $E_{q_{1}, 1}\left(-\alpha_{1} t^{q_{1}}\right)$ and $E_{q_{1}, q_{1}+1}\left(-\alpha_{1} t^{q_{1}}\right)$. If $t$ is large enough, then we have the following approximations:

$$
\begin{aligned}
& E_{q_{1}}\left(-\alpha_{1} t^{q_{1}}\right)=E_{q_{1}, 1}\left(-\alpha_{1} t^{q_{1}}\right) \sim-\sum_{k=1}^{\infty} \frac{\alpha_{1}^{-k}(-t)^{-q_{1} k}}{\Gamma\left(1-q_{1} k\right)}, \\
& t^{q_{1}} E_{q_{1}, q_{1}+1}\left(-\alpha_{1} t_{1}^{q}\right) \sim-t^{q_{1}} \sum_{k=1}^{\infty} \frac{\alpha_{1}^{-k}(-t)^{-q_{1} k}}{\Gamma\left(q_{1}+1-q_{1} k\right)} .
\end{aligned}
$$

It is not difficult to see that as $t \rightarrow \infty, E_{q_{1}}\left(-\alpha_{1} t^{q_{1}}\right)$ in Eq. (37) and $t^{q_{1}} E_{q_{1}, q_{1}+1}\left(-\alpha_{1} t^{q_{1}}\right)$ in Eq. (38) tend to 0 and $\frac{1}{\alpha_{1}}$, respectively. Therefore, $G_{1}(t) \rightarrow \frac{\alpha_{6}}{\alpha_{1}}$ as $t \rightarrow \infty$. Applying this 
result to inequality (36), we have the following consequence:

$$
G(t) \rightarrow \frac{\alpha_{6}}{\alpha_{1}} \quad \text { as } t \rightarrow \infty
$$

and it follows that there exists a constant $M>0$ such that $\|G(t)\| \leq M$. From the second equation of model (29), we have

$$
{ }_{C} D_{a}^{q_{2}} I(t)=-\alpha_{2} I(t)+\alpha_{5} G(t-\tau) \leq-\alpha_{2} I(t)+\alpha_{5} M
$$

Let ${ }_{C} D_{a}^{q_{2}} I_{1}(t)=-\alpha_{2} I_{1}(t)+\alpha_{5} M$ and $I_{1}(0)=I(0)$. Applying the same approach as used above to the resulting fractional differential equation, we then obtain

$$
0 \leq I(t) \leq I_{1}(t)=\alpha_{5} M t^{q_{2}} E_{q_{2}, q_{2}+1}\left(-\alpha_{2} t^{q_{2}}\right)+I_{0} E_{q_{2}, 1}\left(-\alpha_{2} t^{q_{2}}\right), \quad \forall t>0,
$$

and

$$
I(t) \rightarrow \frac{\alpha_{5} M}{\alpha_{2}} \text { as } t \rightarrow \infty
$$

The boundedness statements for $G(t)$ and $I(t)$ imply that the solution $(G(t), I(t))$ exists for all $t>0$. This completes the proof.

\section{Main results}

In this section, some explicit conditions for the occurrence of a Hopf bifurcation for Eq. (29) will be established. Using the transformation $x(t)=G(t)-G^{*}, y(t)=I(t)-I^{*}$, Eq. (29) can be converted to

$$
\begin{aligned}
& { }_{C} D_{a}^{q_{1}} x(t)=-\alpha_{1}\left(x(t)+G^{*}\right)-\frac{\alpha_{3}\left(y(t)+I^{*}\right)\left(x(t)+G^{*}\right)}{\left(\alpha_{4}\left(x(t)+G^{*}\right)+1\right)}+\alpha_{6}, \\
& { }_{C} D_{a}^{q_{2}} y(t)=-\alpha_{2}\left(y(t)+I^{*}\right)+\alpha_{5}\left(x(t-\tau)+G^{*}\right) .
\end{aligned}
$$

Then the linearization of system (43) at the origin leads to

$$
\begin{aligned}
& { }_{C} D_{a}^{q_{1}} x(t)=-m_{1} x(t)-m_{2} y(t), \\
& { }_{C} D_{a}^{q_{2}} y(t)=-\alpha_{2} y(t)+\alpha_{5} x(t-\tau),
\end{aligned}
$$

where

$$
m_{1}=\alpha_{1}+\frac{\alpha_{3} I^{*}}{\left(\alpha_{4} G^{*}+1\right)^{2}}, \quad m_{2}=\frac{\alpha_{3} G^{*}}{\left(\alpha_{4} G^{*}+1\right)} .
$$

The characteristic equation of system (44) can be obtained as

$$
\left|\begin{array}{cc}
s^{q_{1}}+m_{1} & m_{2} \\
-\alpha_{5} e^{-s \tau} & s^{q_{2}}+\alpha_{2}
\end{array}\right|=0,
$$

or equivalently as

$$
s^{q_{1}+q_{2}}+\alpha_{2} s^{q_{1}}+m_{1} s^{q_{2}}+m_{1} \alpha_{2}+m_{2} \alpha_{5} \mathrm{e}^{-s \tau}=0 .
$$


Assume that $s=i \omega=\omega\left(\cos \frac{\pi}{2}+i \sin \frac{\pi}{2}\right)$ is a root of Eq. (47) when $\omega>0$. Substituting $s$ into Eq. (47) and then separating the real and imaginary parts of it, we obtain

$$
\begin{aligned}
& m_{2} \alpha_{5} \cos (\omega \tau)+\omega^{q_{1}+q_{2}} \cos \left(\frac{\left(q_{1}+q_{2}\right) \pi}{2}\right) \\
& +\omega^{q_{1}} \alpha_{2} \cos \left(\frac{q_{1} \pi}{2}\right)+m_{1} \omega^{q_{2}} \cos \left(\frac{q_{2} \pi}{2}\right)+m_{1} \alpha_{2}=0, \\
& -m_{2} \alpha_{5} \sin (\omega \tau)+\omega^{q_{1}+q_{2}} \sin \left(\frac{\left(q_{1}+q_{2}\right) \pi}{2}\right) \\
& +\omega^{q_{1}} \alpha_{2} \sin \left(\frac{q_{1} \pi}{2}\right)+m_{1} \omega^{q_{2}} \sin \left(\frac{q_{2} \pi}{2}\right)=0 .
\end{aligned}
$$

Utilizing Eq. (48) and through direct calculation, we get the following relations:

$$
\begin{aligned}
\cos \omega \tau & =-\frac{\omega^{q_{1}} \alpha_{2} \cos \left(\frac{q_{1} \pi}{2}\right)+m_{1} \omega^{q_{2}} \cos \left(\frac{q_{2} \pi}{2}\right)+\omega^{q_{1}+q_{2}} \cos \left(\frac{\left(q_{1}+q_{2}\right) \pi}{2}\right)+m_{1} \alpha_{2}}{m_{2} \alpha_{5}} \\
& =G_{1}(\omega), \\
\sin \omega \tau & =\frac{\omega^{q_{1}} \alpha_{2} \sin \left(\frac{q_{1} \pi}{2}\right)+m_{1} \omega^{q_{2}} \sin \left(\frac{q_{2} \pi}{2}\right)+\omega^{q_{1}+q_{2}} \sin \left(\frac{\left(q_{1}+q_{2}\right) \pi}{2}\right)}{m_{2} \alpha_{5}}=G_{2}(\omega) .
\end{aligned}
$$

Using Eq. (49) with the fact that $\cos ^{2} \omega \tau+\sin ^{2} \omega \tau=1$, we then have

$$
G_{1}^{2}(\omega)+G_{2}^{2}(\omega)=1
$$

It is assumed that Eq. (50) admits at least one positive real root, denoted by $\omega_{0}$, and we call it the critical frequency. From $\cos \omega \tau=G_{1}(\omega)$, we define

$$
\tau_{j}=\frac{1}{\omega_{0}}\left(\arccos G_{1}\left(\omega_{0}\right)+2 j \pi\right), \quad j=0,1,2, \ldots
$$

Therefore, the bifurcation point of the system, associated with $\omega_{0}$, is

$$
\tau_{0}=\min \left\{\tau_{j}\right\}, \quad j=0,1,2, \ldots
$$

To derive the condition for the occurrence of a Hopf bifurcation, we announce the following hypothesis:

$$
\text { (H1) } \frac{R_{1} R_{2}+I_{1} I_{2}}{R_{2}^{2}+I_{2}^{2}} \neq 0,
$$

where

$$
\begin{aligned}
R_{1}= & m_{2} \alpha_{5} \omega_{0}^{2} \cos \left(\omega_{0} \tau_{0}\right), \quad I_{1}=-m_{2} \alpha_{5} \omega_{0}^{2} \sin \left(\omega_{0} \tau_{0}\right), \\
R_{2}= & \omega_{0} \tau_{0} m_{2} \alpha_{5} \sin \left(\omega_{0} \tau_{0}\right)-\omega_{0}^{q_{1}} q_{1} \alpha_{2} \cos \left(\frac{q_{1} \pi}{2}\right)-\omega_{0}^{q_{2}} q_{2} m_{1} \cos \left(\frac{q_{2} \pi}{2}\right) \\
& -\omega_{0}^{q_{1}+q_{2}} q_{1} \cos \left(\frac{\left(q_{1}+q_{2}\right) \pi}{2}\right)-\omega_{0}^{q_{1}+q_{2}} q_{2} \cos \left(\frac{\left(q_{1}+q_{2}\right) \pi}{2}\right),
\end{aligned}
$$




$$
\begin{aligned}
I_{2}= & \omega_{0} \tau_{0} m_{2} \alpha_{5} \cos \left(\omega_{0} \tau_{0}\right)-\omega_{0}^{q_{1}} q_{1} \alpha_{2} \sin \left(\frac{q_{1} \pi}{2}\right)-\omega_{0}^{q_{2}} q_{2} m_{1} \sin \left(\frac{q_{2} \pi}{2}\right) \\
& -\omega_{0}^{q_{1}+q_{2}} q_{1} \sin \left(\frac{\left(q_{1}+q_{2}\right) \pi}{2}\right)-\omega^{q_{1}+q_{2}} q_{2} \sin \left(\frac{\left(q_{1}+q_{2}\right) \pi}{2}\right) .
\end{aligned}
$$

Lemma 5.1 Let $s(\tau)=\gamma(\tau)+i \omega(\tau)$ be the root of Eq. (47). It is obvious to see that $\gamma\left(\tau_{k}\right)=0$, $\omega\left(\tau_{k}\right)=\omega_{0}$ when $\tau=\tau_{k}$, then the condition holds

$$
\operatorname{Re}\left[\frac{d s}{d \tau}\right]_{\tau=\tau_{0}, \omega=\omega_{0}} \neq 0 .
$$

Proof Differentiating both sides of Eq. (47) with respect to $\tau$, we attain

$$
s^{q_{1}+q_{2}-1}\left(q_{1}+q_{2}\right) \frac{d s}{d \tau}+s^{q_{1}-1} q_{1} \alpha_{2} \frac{d s}{d \tau}+s^{q_{2}-1} q_{2} m_{1} \frac{d s}{d \tau}+m_{2} \alpha_{5} e^{-s \tau}\left(-\frac{d s}{d \tau} \tau-s\right)=0 .
$$

Then we have

$$
\frac{d s}{d \tau}=\frac{e^{-s \tau} s^{2} m_{2} \alpha_{5}}{-e^{-s \tau} s \tau m_{2} \alpha_{5}+s^{q_{1}} q_{1} \alpha_{2}+s^{q_{2}} q_{2} m_{1}+s^{q_{1}+q_{2}} q_{1}+s^{q_{1}+q_{2}} q_{2}}=\frac{M(s)}{N(s)} .
$$

By straightforward computation, we can deduce

$$
\operatorname{Re}\left[\frac{d s}{d \tau}\right]_{\tau=\tau_{0}, \omega=\omega_{0}}=\frac{R_{1} R_{2}+I_{1} I_{2}}{R_{2}^{2}+I_{2}^{2}}
$$

where $R_{1}, I_{1}$ are the real and imaginary parts of $M(s)$ evaluated at $\tau=\tau_{0}, \omega=\omega_{0}$ and $R_{2}, I_{2}$ are the real and imaginary parts of $N(s)$ evaluated at $\tau=\tau_{0}, \omega=\omega_{0}$ as defined in Eq. (53). Obviously, hypothesis (H1) indicates that the transversality condition is satisfied. This completes the proof.

Lemma 5.2 The positive equilibrium point $\left(G^{*}, I^{*}\right)$ of the fractional-order system (29) is locally asymptotically stable when $\tau=0$.

Proof If there is no delay, i.e., $\tau=0$, Eq. (47) becomes

$$
\lambda^{2}+\left(\alpha_{2}+m_{1}\right) \lambda+\left(\alpha_{2} m_{1}+\alpha_{5} m_{2}\right)=0 .
$$

The roots of the above characteristic equation are

$$
\begin{aligned}
& \lambda_{+}=\frac{-\left(\alpha_{2}+m_{1}\right)+\sqrt{\left(\alpha_{2}+m_{1}\right)^{2}-4\left(\alpha_{2} m_{1}+\alpha_{5} m_{2}\right)}}{2}, \\
& \lambda_{-}=\frac{-\left(\alpha_{2}+m_{1}\right)-\sqrt{\left(\alpha_{2}+m_{1}\right)^{2}-4\left(\alpha_{2} m_{1}+\alpha_{5} m_{2}\right)}}{2} .
\end{aligned}
$$

It is not difficult to see that $\operatorname{Re}\left(\lambda_{ \pm}\right)<0$. Hence, the positive equilibrium point $\left(G^{*}, I^{*}\right)$ of fractional-order system (29) is locally asymptotically stable when $\tau=0$.

Remark 5.1 In agreement with the theory of stability for fractional-order systems, the real parts of all roots of the characteristic equation in Eq. (57) are negative, which can assure 
that all the roots of Eq. (57) satisfy $\left|\arg \left(\lambda_{i}\right)\right|>\frac{q_{i} \pi}{2}(i=1,2)$, and so Lemma 5.2 may still hold for system (29).

Next we can establish the existence of a Hopf bifurcation of fractional-order system (29) as follows.

Theorem 5.1 Suppose that (H1) holds for system (29), then the following results hold:

(i) The equilibrium point $\left(G^{*}, I^{*}\right)$ of system (29) is locally asymptotically stable for $\tau \in\left[0, \tau_{0}\right)$, and unstable when $\tau>\tau_{0}$.

(ii) System (29) displays a Hopf bifurcation at the equilibrium point $\left(G^{*}, I^{*}\right)$ when $\tau=\tau_{0}$. In other words, near $\tau=\tau_{0}$, there is a branch of periodic solutions bifurcating from the equilibrium point $\left(G^{*}, I^{*}\right)$.

\section{Numerical results}

In this section, we will provide four numerical examples to verify our theoretical results obtained in Sect. 5. The numerical solutions for system (29) presented in this section are simulated using the Adams-Bashforth-Moulton type predictor-corrector scheme described in Sect. 2 with the step-size $h=0.01$. In addition, the parameter values and the initial conditions $[8,9]$ used in the simulations are as follows: $a_{1}=0.0001, a_{2}=0.1262$, $a_{3}=5.64 \times 10^{-5}, a_{4}=0.01, a_{5}=0.074, a_{6}=1.93$ and $G(0)=87, I(0)=60$. Using the relation expressed in (28) and the fractional time constant $\beta=1$, we have the following information for our simulations:

$$
\begin{aligned}
& \alpha_{3}=5.64 \times 10^{-5}, \quad \alpha_{4}=0.01, \quad \alpha_{5}=0.074, \\
& \alpha_{6}=1.93, \quad G(0)=87, \quad I(0)=60,
\end{aligned}
$$

but the values of $\alpha_{1}$ and $\alpha_{2}$, which depend upon the fractional orders $q_{1}$ and $q_{2}$, will be computed later.

In order to see the impact of the time delay $\tau$ on the dynamics of system (29), the bifurcation point $\tau_{0}$ is required to be calculated. We employ four sets of the fractional orders $q_{1}$ and $q_{2}$ as shown in Table 2 to perform the dynamics of the system. Also, the values of $\alpha_{1}$, $\alpha_{2}, \omega_{0}$, and $\tau_{0}$, which can be computed using Eqs. (28), (50), (51), and the values of $q_{1}, q_{2}$ from the mentioned sets are included in Table 2. The following four numerical simulations using the information in Table 2 are discussed below.

Case I: We use the values of $q_{1}, q_{2}, \alpha_{1}$, and $\alpha_{2}$ from Set 1 in Table 2 and the information (58) to simulate system (29). The system is reduced into the regular integerorder system. From (30), the positive equilibrium point of the system for this case is $\left(G^{*}, I^{*}\right)=(650.592,381.488)$. From Table 2, we have $\omega_{0}=0.002825$ and $\tau_{0}=607.919$. This value of $\tau_{0}$ for this integer-order case is in the acceptable range for obtaining

Table 2 The values of the fractional orders $q_{1}, q_{2}$ and the computed values of $\alpha_{1}, \alpha_{2}, \omega_{0}, \tau_{0}$ for the numerical simulations of system (29)

\begin{tabular}{llllllr}
\hline Set number & $q_{1}$ & $q_{2}$ & $\alpha_{1}$ & $\alpha_{2}$ & $\omega_{0}$ & \multicolumn{1}{c}{$\tau_{0}$} \\
\hline Set 1 & 1 & 1 & 0.0001 & 0.1262 & 0.002825 & 607.919 \\
Set 2 & 0.92 & 0.92 & 0.0002 & 0.1489 & 0.001382 & 1365.600 \\
Set 3 & 0.92 & 0.95 & 0.0002 & 0.1399 & 0.001469 & 1285.910 \\
Set 4 & 0.92 & 0.72 & 0.0002 & 0.2253 & 0.000903 & 2089.425 \\
\hline
\end{tabular}



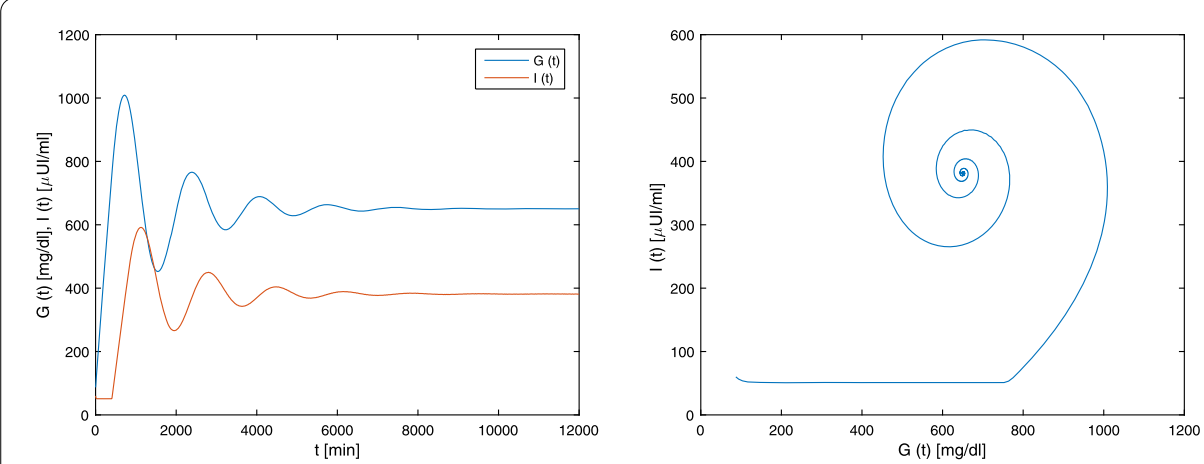

Figure 1 Time series and phase portrait of fractional-order model (29) generated using the information from Set 1 , the parameter values in Eq. (58), and $\tau=400<\tau_{0}=607.919$. The positive equilibrium $\left(G^{*}, 1^{*}\right)=(650.592,381.488)$ of the system is asymptotically stable
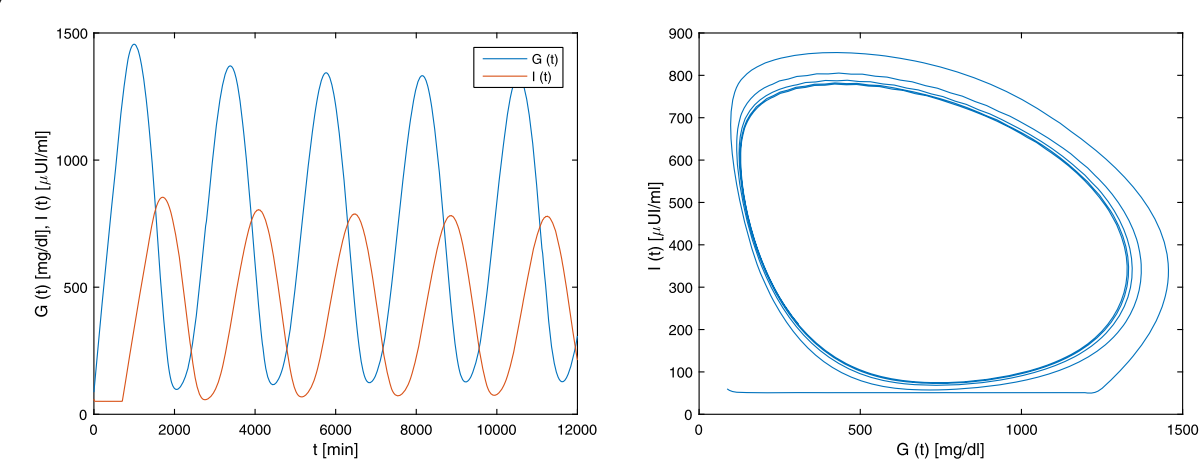

Figure 2 Time series and phase portrait of fractional-order model (29) generated using the information from Set 1 , the parameter values in Eq. (58), and $\tau=700>\tau_{0}=607.919$. A periodic oscillation bifurcates from the equilibrium point $\left(G^{*}, I^{*}\right)=(650.592,381.488)$

sustainable oscillatory solutions as mentioned in [9]. Using Eq. (56), we easily obtain $\operatorname{Re}\left[\frac{d s}{d \tau}\right]_{\tau=\tau_{0}, \omega=\omega_{0}}=0.000001695 \neq 0$. Hence, by means of Theorem 5.1, the positive equilibrium point $\left(G^{*}, I^{*}\right)$ is asymptotically stable when $\tau=400<\tau_{0}$, which is demonstrated in Fig. 1 for $t \in[0,12,000]$. While $\tau=700>\tau_{0}$, this equilibrium point is unstable, and there occurs a Hopf bifurcation at the equilibrium point as illustrated in Fig. 2 for $t \in[0,12,000]$.

Case II: We utilize the values of $q_{1}, q_{2}, \alpha_{1}$, and $\alpha_{2}$ from Set 2 in Table 2 and the information (58) to numerically solve system (29). The system is reduced into the commensurate one with $q_{1}=q_{2}=0.92$. From (30), the positive equilibrium point of the system for this case is $\left(G^{*}, I^{*}\right)=(722.65,359.07)$, and from Table 2, we have $\omega_{0}=0.001382$ and $\tau_{0}=1365.60$. Using Eq. (56), we easily obtain $\operatorname{Re}\left[\frac{d s}{d \tau}\right]_{\tau=\tau_{0}, \omega=\omega_{0}}=3.238532623 \times 10^{-7} \neq 0$. Hence, by Theorem 5.1, the equilibrium point for this case is asymptotically stable when $\tau=1000<\tau_{0}$, which is illustrated in Fig. 3 for $t \in[0,40,000]$. While it is unstable and a Hopf bifurcation emerges when $\tau=1600>\tau_{0}$, as simulated in Fig. 4 for $t \in[0,40,000]$.

Case III: In this case, we utilize the values of $q_{1}, q_{2}, \alpha_{1}$, and $\alpha_{2}$ from Set 3 in Table 2 and the parameter values and the initial conditions expressed in Eq. (58) to numerically simulate system (29). From (30), the positive equilibrium point of the incommensurate fractional-order model with $q_{1}=0.92 q_{2}=0.95$ is $\left(G^{*}, I^{*}\right)=(686.41,362.92)$, and from Table 2, we have $\omega_{0}=0.001469$ and $\tau_{0}=1285.910$. Using Eq. (56), it is not difficult to ob- 

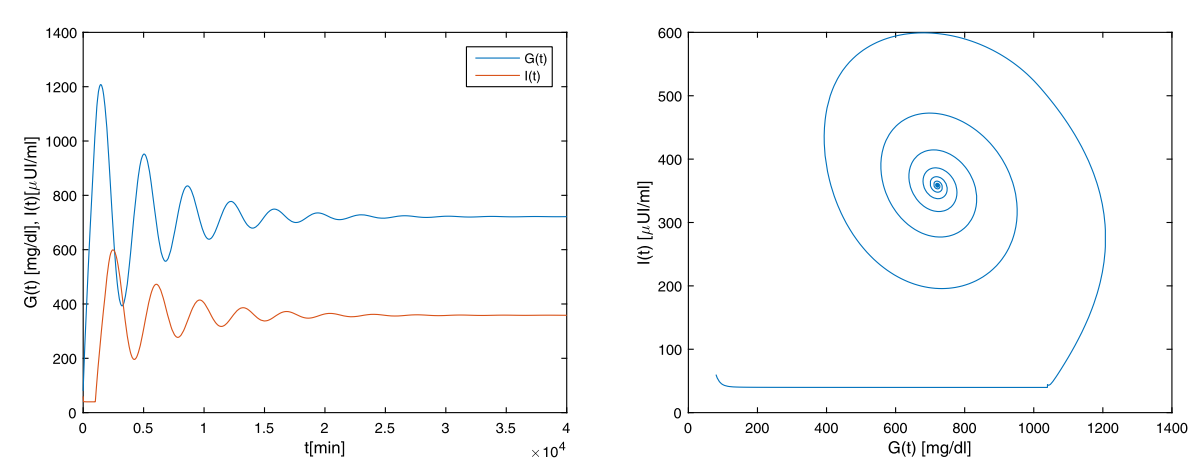

Figure 3 Time series and phase portrait of fractional-order model (29) generated using the information from Set 2, the parameter values in Eq. (58), and $\tau=1000<\tau_{0}=1365.60$. The positive equilibrium $\left(G^{*}, \prime^{*}\right)=(722.65,359.07)$ of the system is asymptotically stable
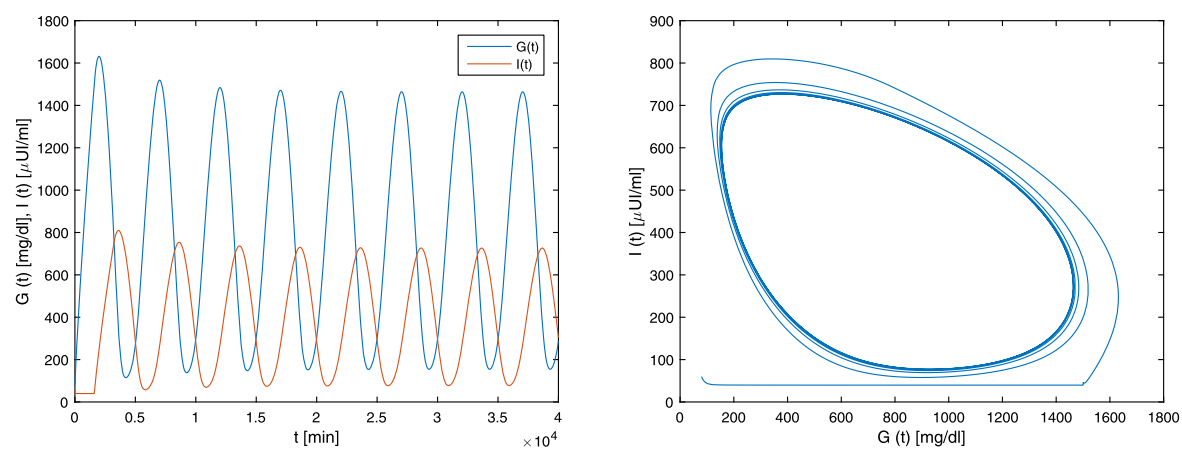

Figure 4 Time series and phase portrait of fractional-order model (29) generated using the information from Set 2 , the parameter values in Eq. (58), and $\tau=1600>\tau_{0}=1365.60$. A periodic oscillation bifurcates from the equilibrium point $\left(G^{*}, I^{*}\right)=(722.65,359.07)$
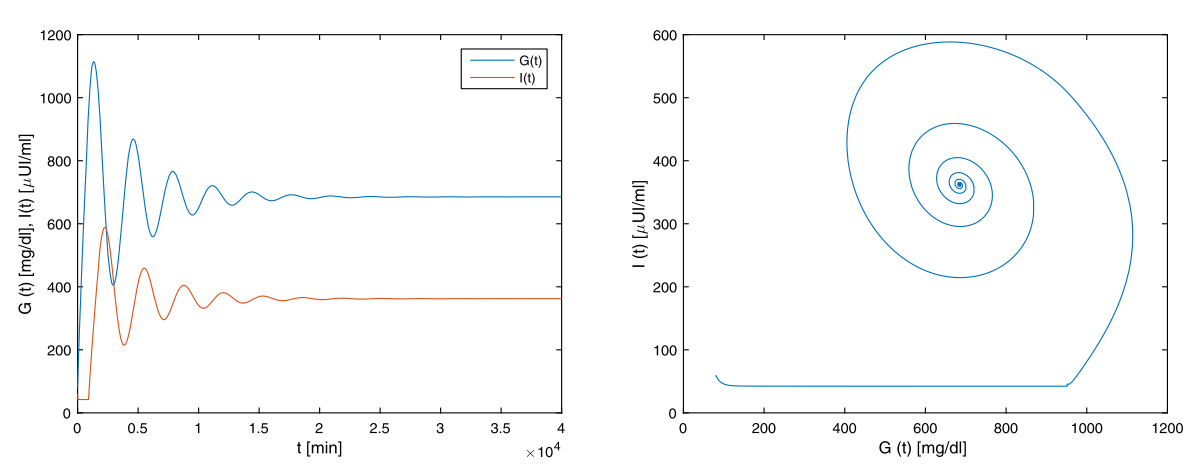

Figure 5 Time series and phase portrait of fractional-order model (29) generated using the information from Set 3 , the parameter values in Eq. (58), and $\tau=900<\tau_{0}=1285.91$. The positive equilibrium $\left(G^{*}, I^{*}\right)=(686.41,362.92)$ of the system is asymptotically stable

tain $\operatorname{Re}\left[\frac{d s}{d \tau}\right]_{\tau=\tau_{0}, \omega=\omega_{0}}=3.650184 \times 10^{-7} \neq 0$. Thus, by Theorem 5.1 , the positive equilibrium point for this case is asymptotically stable when $\tau=900<\tau_{0}$, which is shown in Fig. 5 for $t \in[0,40,000]$. However,it is unstable and a Hopf bifurcation occurs when $\tau=1500>\tau_{0}$, as displayed in Fig. 6 for $t \in[0,40,000]$. 

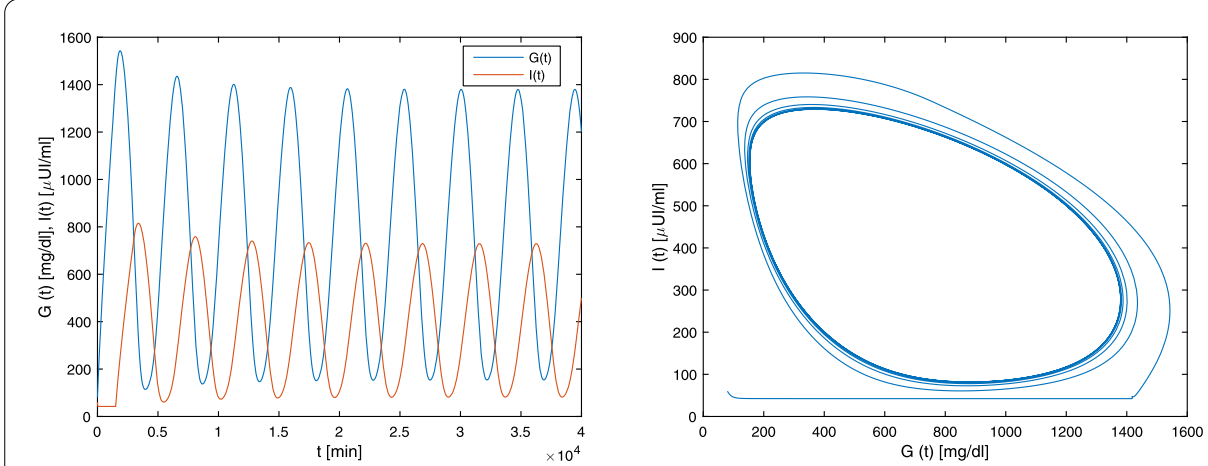

Figure 6 Time series and phase portrait of fractional-order model (29) generated using the information from Set 3 , the parameter values in Eq. (58), and $\tau=1500>\tau_{0}=1285.910$. A periodic oscillation bifurcates from the equilibrium point $\left(G^{*}, I^{*}\right)=(686.41,362.92)$
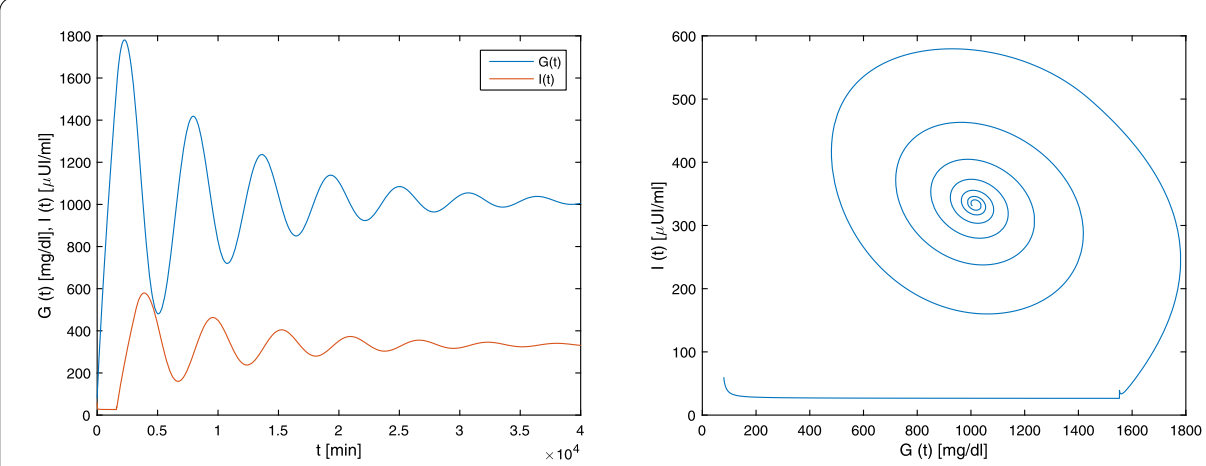

Figure 7 Time series of fractional-order model (29) generated using the information from Set 4, the parameter values in Eq. (58), and $\tau=1400<\tau_{0}=2089.425$. The positive equilibrium $\left(G^{*}, I^{*}\right)=(1018.09,334.39)$ of the system is asymptotically stable

Case IV: Employing the values of $q_{1}, q_{2}, \alpha_{1}$, and $\alpha_{2}$ from Set 4 in Table 2 and the information (58) for system (29), we perform numerical solutions of the obtained incommensurate fractional-order system with $q_{1}=0.92 q_{2}=0.72$. From (30), the positive equilibrium point of the system for this case is $\left(G^{*}, I^{*}\right)=(1018.09,334.39)$, and we obtain $\omega_{0}=0.000903$ and $\tau_{0}=2089.425$ as expressed in Table 2. Using Eq. (56), we obtain $\operatorname{Re}\left[\frac{d s}{d \tau}\right]_{\tau=\tau_{0}, \omega=\omega_{0}}=1.373511 \times 10^{-7} \neq 0$. According to Theorem 5.1 , the positive equilibrium point for this case is asymptotically stable when $\tau=1400<\tau_{0}$. The graphical result is shown in Fig. 7 for $t \in[0,40,000]$. Actually, this equilibrium point is asymptotically stable when $\tau \in[0,2089.425)$. However,it is unstable and a Hopf bifurcation emerges when $\tau=2400>\tau_{0}$, as displayed in Fig. 8 for $t \in[0,40,000]$.

In the following part, we will discuss the different effects of each of the fractional orders on bifurcation for system (29). The parameter values, which are used in our experiments, can be calculated using relation (28) with the fractional time constant $\beta=1$. For the sake of convenience, we fix one of the fractional orders and vary the other one to investigate the effects of the altered one on the bifurcation of the system. Here the experiments are as follows.

Experiment I: We set $q_{1}=0.92$, then we observe the effects of $q_{2}$ on bifurcation for system (29). In this case, we easily calculate the corresponding critical frequency $\omega_{0}$ and bi- 

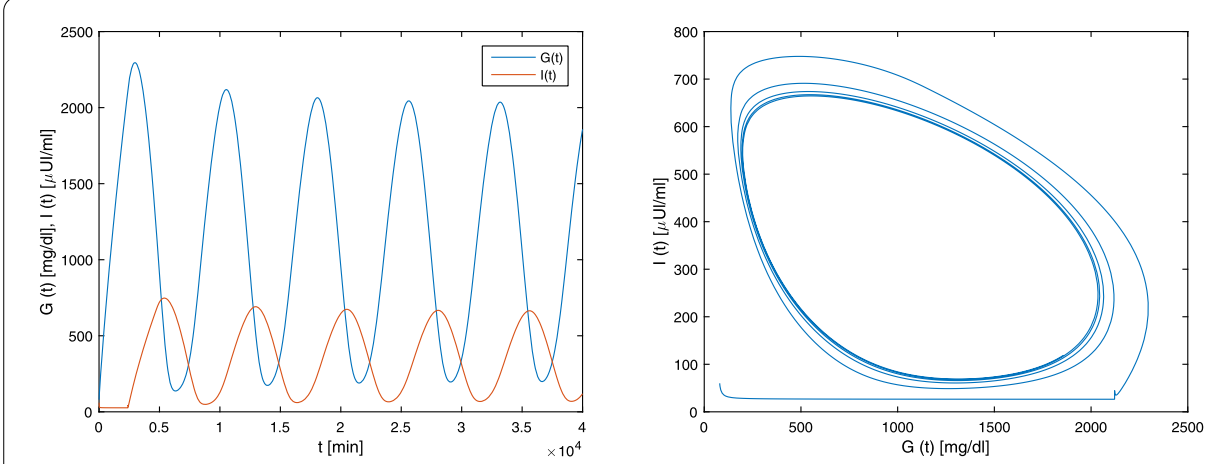

Figure 8 Time series of fractional-order model (29) generated using the information from Set 4, the parameter values in Eq. (58), and $\tau=2400>\tau_{0}=2089.425$. A periodic oscillation bifurcates from the equilibrium point $\left(G^{*}, I^{*}\right)=(1018.09,334.39)$

Table 3 The effect of $q_{2}$ on the values of $\omega_{0}$ and $\tau_{0}$ for system (29) when $q_{1}=0.92$

\begin{tabular}{lll}
\hline Fractional order $q_{2}$ & Critical frequency $\omega_{0}$ & Bifurcation point $\tau_{0}$ \\
\hline 0.65 & 0.000770 & 2454.599 \\
0.7 & 0.000863 & 2186.146 \\
0.72 & 0.000903 & 2089.425 \\
0.75 & 0.000964 & 1954.355 \\
0.79 & 0.001052 & 1790.990 \\
0.83 & 0.001146 & 1644.418 \\
0.85 & 0.001195 & 1576.753 \\
0.88 & 0.001272 & 1481.622 \\
0.92 & 0.001382 & 1365.600 \\
0.95 & 0.001469 & 1285.910 \\
1 & 0.001624 & 1165.495 \\
\hline
\end{tabular}

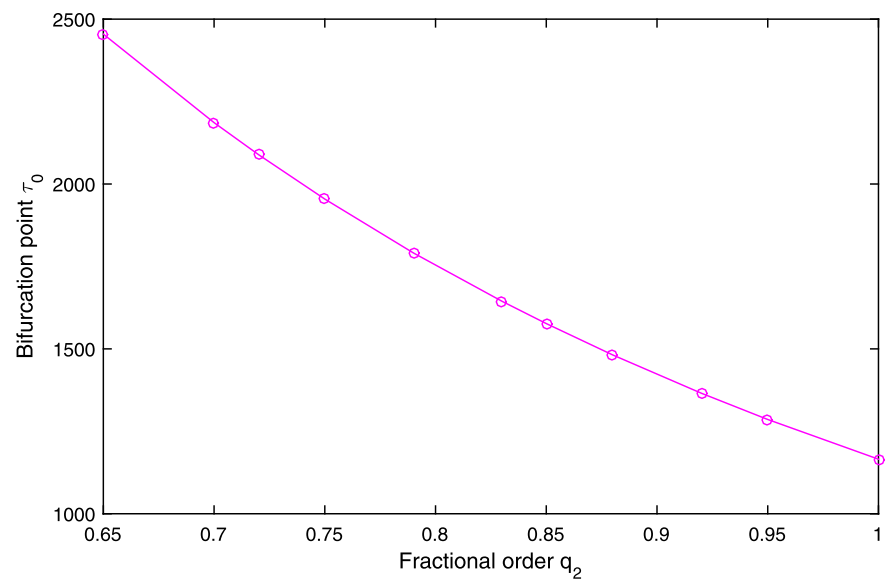

Figure 9 Graph of bifurcation point $\tau_{0}$ versus the order $q_{2}$ for system (29) when $q_{1}$ is fixed at 0.92

furcation point $\tau_{0}$ as $q_{2}$ is varied. The computed values of $\omega_{0}, \tau_{0}$ are listed in Table 3 . It can be easily concluded from Table 3 and Fig. 9 that the bifurcation occurs in advance as $q_{2}$ increases.

Experiment II: We set $q_{2}=0.95$, then we study the effects of $q_{1}$ on bifurcation for system (29). In this case, we can compute the corresponding critical frequency $\omega_{0}$ and bifurcation 
Table 4 The effect of $q_{1}$ on the values of $\omega_{0}$ and $\tau_{0}$ for system (29) when $q_{2}=0.95$

\begin{tabular}{llc}
\hline Fractional order $q_{1}$ & Critical frequency $\omega_{0}$ & Bifurcation point $\tau_{0}$ \\
\hline 0.73 & 0.000119 & $21,921.541$ \\
0.75 & 0.000193 & $12,916.565$ \\
0.76 & 0.000235 & $10,368.426$ \\
0.78 & 0.000330 & 7083.487 \\
0.81 & 0.000502 & 4416.125 \\
0.85 & 0.000789 & 2639.034 \\
0.88 & 0.001050 & 1897.274 \\
0.92 & 0.001469 & 1285.910 \\
0.95 & 0.001839 & 988.433 \\
0.98 & 0.002261 & 774.341 \\
1 & 0.002574 & 663.853 \\
\hline
\end{tabular}

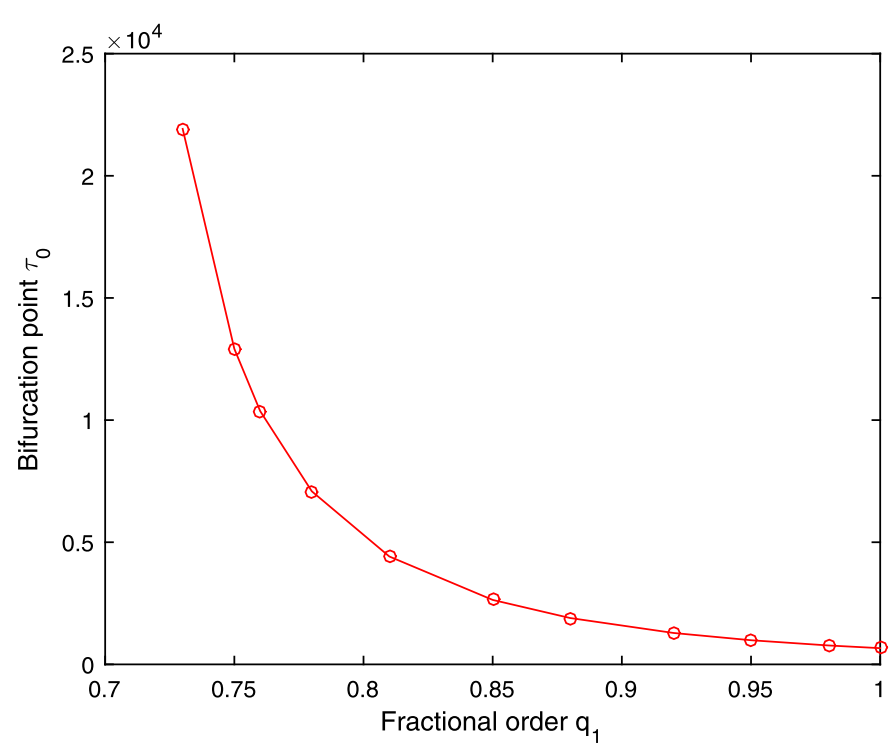

Figure 10 Graph of bifurcation point $\tau_{0}$ versus the order $q_{1}$ for system (29) when $q_{2}$ is fixed at 0.95

point $\tau_{0}$ as $q_{1}$ is changed. The obtained values of $\omega_{0}, \tau_{0}$ are described in Table 4 . It can be easily observed from Table 4 and Fig. 10 that the bifurcation emerges ahead of time as $q_{1}$ increases.

\section{Conclusion}

In this article, we have proposed the delayed fractional differential equation model for glucose-insulin interaction using the Caputo fractional derivative with incommensurate orders. The unique positive equilibrium point of the system has been discovered. We have also established the conditions for stability of the equilibrium point and Hopf bifurcation. It has been found from the stability analysis that the time delay $\tau$ in the system has significant influence on the stability. In fact, stability regions of fractional-order model (29) can be adjusted by varying the fractional orders of the model as simulated in experiments I and II of Sect. 6. Furthermore, the Hopf bifurcation has occurred prematurely as one of the fractional orders increases while the other is fixed. The numerical examples obtained using the Adams-Bashforth-Moulton type predictor-corrector scheme have verified our main theoretical results. 
Acknowledgements

The authors are grateful to the referees for their careful reading of this paper and valuable comments.

\section{Funding}

This research was partially funded by the Centre of Excellence in Mathematics, the Commission on Higher Education, Thailand and the Graduate College, King Mongkut's University of Technology North Bangkok, Thailand.

\section{Availability of data and materials}

Not applicable.

\section{Competing interests}

The authors declare that they have no competing interests.

\section{Authors' contributions}

All authors worked together to produce the results, and they all read and approved the final manuscript.

\section{Publisher's Note}

Springer Nature remains neutral with regard to jurisdictional claims in published maps and institutional affiliations.

\section{Received: 4 February 2019 Accepted: 25 July 2019 Published online: 06 August 2019}

\section{References}

1. Alexander, M.E., Mathur, R., Moghadas, S.M., Shivakumar, P.N., et al.: Modelling the effect of CSIl on the control of glucose concentration in type 1 diabetes. Appl. Math. Comput. 187(2), 1476-1483 (2007)

2. Huang, C.-N., Chou, W.-C., Lin, L.-Y., Peng, C.-C., Chyau, C.-C., Chen, K.-C., Peng, R.Y.: First phase release coefficient of insulin in subjects with normal glucose tolerance on glucose infusion analyzed by computer simulation. Biosystems 91(1), 146-157 (2008)

3. Chávez, I.Y.S., Morales-Menéndez, R., Chapa, S.O.M.: Glucose optimal control system in diabetes treatment. Appl. Math. Comput. 209(1), 19-30 (2009)

4. Duarte, J., Januário, C., Martins, N.: Topological entropy and the controlled effect of glucose in the electrical activity of pancreatic $\beta$-cells. Phys. D, Nonlinear Phenom. 238(21), 2129-2137 (2009)

5. Giang, D.V., Lenbury, Y., De Gaetano, A., Palumbo, P.: Delay model of glucose-insulin systems: global stability and oscillated solutions conditional on delays. J. Math. Anal. Appl. 343(2), 996-1006 (2008)

6. Bergman, R.N., Phillips, L.S., Cobelli, C.: Physiologic evaluation of factors controlling glucose tolerance in man: measurement of insulin sensitivity and beta-cell glucose sensitivity from the response to intravenous glucose. J. Clin. Invest. 68(6), 1456-1467 (1981)

7. Pacini, G., Bergman, R.N.: Minmod: a computer program to calculate insulin sensitivity and pancreatic responsivity from the frequently sampled intravenous glucose tolerance test. Comput. Methods Programs Biomed. 23(2), 113-122 (1986)

8. De Gaetano, A., Arino, O.: Mathematical modelling of the intravenous glucose tolerance test. J. Math. Biol. 40(2), $136-168(2000)$

9. Li, J., Kuang, Y., Li, B.: Analysis of IVGTT glucose-insulin interaction models with time delay. Discrete Contin. Dyn. Syst., Ser. B 1(1), 103-124 (2001)

10. Bergman, R.N., Ider, Y.Z., Bowden, C.R., Cobelli, C.: Quantitative estimation of insulin sensitivity. Am. J. Physiol: Endocrinol. Metab. 236(6), 667 (1979)

11. Sturis, J., Polonsky, K.S., Mosekilde, E., Van Cauter, E.: Computer model for mechanisms underlying ultradian oscillations of insulin and glucose. Am. J. Physiol: Endocrinol. Metab. 260(5), 801-809 (1991)

12. Engelborghs, K., Lemaire, V., Belair, J., Roose, D.: Numerical bifurcation analysis of delay differential equations arising from physiological modeling. J. Math. Biol. 42(4), 361-385 (2001)

13. Rakkiyappan, R., Udhayakumar, K., Velmurugan, G., Cao, J., Alsaedi, A.: Stability and Hopf bifurcation analysis of fractional-order complex-valued neural networks with time delays. Adv. Differ. Equ. 2017(1), 225 (2017)

14. Abdelaziz, M.A., Ismail, A.I., Abdullah, F.A., Mohd, M.H.: Bifurcations and chaos in a discrete SI epidemic model with fractional order. Adv. Differ. Equ. 2018(1), 44 (2018)

15. Sun, H., Zhang, Y., Baleanu, D., Chen, W., Chen, Y.: A new collection of real world applications of fractional calculus in science and engineering. Commun. Nonlinear Sci. Numer. Simul. 64, 213-231 (2018)

16. Zou, Q., Jin, Q., Zhang, R.: Design of fractional order predictive functional control for fractional industrial processes. Chemom. Intell. Lab. Syst. 152, 34-41 (2016)

17. Podlubny, I.: Fractional Differential Equations: An Introduction to Fractional Derivatives, Fractional Differential Equations, to Methods of Their Solution and Some of Their Applications, vol. 198. Elsevier, Amsterdam (1998)

18. Almeida, R.: A Caputo fractional derivative of a function with respect to another function. Commun. Nonlinear Sci. Numer. Simul. 44, 460-481 (2017)

19. Scherer, R., Kalla, S.L., Tang, Y., Huang, J.: The Grünwald-Letnikov method for fractional differential equations. Comput. Math. Appl. 62(3), 902-917 (2011)

20. Ertürk, V.S., Zaman, G., Momani, S.: A numeric-analytic method for approximating a giving up smoking model containing fractional derivatives. Comput. Math. Appl. 64(10), 3065-3074 (2012)

21. Cho, Y., Kim, I., Sheen, D.: A fractional-order model for minmod millennium. Math. Biosci. 262, 36-45 (2015)

22. Carvalho, A., Pinto, C.M.: A delay fractional order model for the co-infection of malaria and HIV/AIDS. Int. J. Dyn. Control 5(1), 168-186 (2017)

23. Huang, C., Cao, J., Xiao, M., Alsaedi, A., Alsaadi, F.E.: Controlling bifurcation in a delayed fractional predator-prey system with incommensurate orders. Appl. Math. Comput. 293, 293-310 (2017) 
24. Lekdee, N., Sirisubtawee, S., Koonprasert, S.: Exact solutions and numerical comparison of methods for solving fractional-order differential systems. In: IMECS 2018. Lecture Notes in Engineering and Computer Science, vol. 2 pp. 459-466 (2018)

25. Moore, E.J., Sirisubtawee, S., Koonprasert, S.: A Caputo-Fabrizio fractional differential equation model for HIV/AIDS with treatment compartment. Adv. Differ. Equ. 2019(1), 200 (2019)

26. Carvalho, A.R., Pinto, C.M., Baleanu, D.: HIV/HCV coinfection model: a fractional-order perspective for the effect of the HIV viral load. Adv. Differ. Equ. 2018(1), 2 (2018)

27. Ucar, E., Özdemir, N., Altun, E.: Fractional order model of immune cells influenced by cancer cells. Math. Model. Nat. Phenom. 14(3), 308 (2019)

28. Singh, J., Kumar, D., Baleanu, D.: On the analysis of fractional diabetes model with exponential law. Adv. Differ. Equ. 2018(1), 231 (2018)

29. MacRury, S., Lowe, G.: Blood rheology in diabetes mellitus. Diabet. Med. 7(4), 285-291 (1990)

30. Moan, A., Nordby, G., Os, I., Birkeland, K.I., Kjeldsen, S.E.: Relationship between hemorrheologic factors and insulin sensitivity in healthy young men. Metabolism 43(4), 423-427 (1994)

31. Pérez-Martin, A., Dumortier, M., Pierrisnard, E., Raynaud, E., Mercier, J., Brun, J.: Multivariate analysis of relationships between insulin sensitivity and blood rheology: is plasma viscosity a marker of insulin resistance? Clin. Hemorheol. Microcirc. 25(3-4), 91-103 (2001)

32. Mahata, A., Mondal, S.P., Alam, S., Roy, B.: Mathematical model of glucose-insulin regulatory system on diabetes mellitus in fuzzy and crisp environment. Ecol. Genet. Genomics 2, 25-34 (2017)

33. Farman, M., Saleem, M.U., Tabassum, M., Ahmad, A., Ahmad, M.: A linear control of composite model for glucose insulin glucagon pump. Ain Shams Eng. J. (2019). https://doi.org/10.1016/j.asej.2019.04.001

34. Shabestari, P.S., Panahi, S., Hatef, B., Jafari, S., Sprott, J.C.: A new chaotic model for glucose-insulin regulatory system. Chaos Solitons Fractals 112, 44-51 (2018)

35. Tolić, I.M., Mosekilde, E., Sturis, J.: Modeling the insulin-glucose feedback system: the significance of pulsatile insulin secretion. J. Theor. Biol. 207(3), 361-375 (2000)

36. Sakulrang, S., Moore, E.J., Sungnul, S., de Gaetano, A.: A fractional differential equation model for continuous glucose monitoring data. Adv. Differ. Equ. 2017(1), 150 (2017)

37. Derouich, M., Boutayeb, A.: The effect of physical exercise on the dynamics of glucose and insulin. J. Biomech. 35(7), 911-917 (2002)

38. Kilbas, A.A.A., Srivastava, H.M., Trujillo, J.J.: Theory and Applications of Fractional Differential Equations, vol. 204. Elsevier, Amsterdam (2006)

39. Ding, Y., Ye, H.: A fractional-order differential equation model of HIV infection of cd4+ t-cells. Math. Comput. Model. 50(3-4), 386-392 (2009)

40. Li, Y., Chen, Y., Podlubny, I.: Mittag-Leffler stability of fractional order nonlinear dynamic systems. Automatica 45(8), 1965-1969 (2009)

41. Zhou, X., Wu, Z., Wang, Z., Zhou, T.: Stability and Hopf bifurcation analysis in a fractional-order delayed paddy ecosystem. Adv. Differ. Equ. 2018(1), 315 (2018)

42. Bhalekar, S., Daftardar-Gejji, V.: A predictor-corrector scheme for solving nonlinear delay differential equations of fractional order. J. Fract. Calc. Appl. 1(5), 1-9 (2011)

43. Magin, R., Feng, X., Baleanu, D.: Solving the fractional order Bloch equation. Concepts Magn. Reson., Part A 34(1), 16-23 (2009)

\section{Submit your manuscript to a SpringerOpen ${ }^{\circ}$ journal and benefit from:}

- Convenient online submission

- Rigorous peer review

- Open access: articles freely available online

- High visibility within the field

- Retaining the copyright to your article

Submit your next manuscript at $\boldsymbol{s p r i n g e r o p e n . c o m ~}$ 